\title{
ISOSPECTRAL RIEMANNIAN METRICS AND POTENTIALS
}

\author{
DENNIS M. DETURCK AND CAROLYN S. GORDON
}

For $(M, g)$ a compact Riemannian manifold, we consider the spectra of the Laplace-Beltrami operator and of the Schrödinger operator "Laplacian plus potential" acting on $L^{2}(M, g)$. Two Riemannian manifolds are said to be isospectral if their associated Laplacians have the same spectra, and two potentials on the same Riemannian manifold are said to be isospectral if the associated Schrödinger operators have the same spectra. Generalizing methods of Sunada $[\mathbf{S}]$ and Brooks $[\mathbf{B}]$, we give a fairly general technique for constructing isospectral metrics and potentials. In the case of metrics, our method unifies the various methods used previously by numerous authors to construct isospectral metrics and allows us to construct many new examples; in the case of potentials, we obtain many new examples of continuous families of isospectral, noncongruent potentials.

In all the examples known of isospectral closed manifolds, the manifolds have a common Riemannian cover. Thus, they are of the form $\left(\Gamma_{i} \backslash M, g\right)$, $i=1,2$, where $(M, g)$ is a Riemannian manifold and each $\Gamma_{i}$ is a discrete group acting freely and properly discontinuously by isometries on $(M, g)$. Moreover, with one exception (noted below), there exists a bijection between $\Gamma_{1}$ and $\Gamma_{2}$ such that corresponding elements are conjugate in the full isometry group $I(M)$. It is not known whether these conditions are sufficient for the quotient manifolds to be isospectral. Sunada $[\mathbf{S}]$ proved under these conditions that if $\Gamma_{1}$ and $\Gamma_{2}$ are both contained in a finite subgroup $G$ of $I(M)$ which acts freely on $M$ and if corresponding elements of $\Gamma_{1}$ and $\Gamma_{2}$ are conjugate within $G$, then the manifolds are isospectral. (Of course, if the groups $\Gamma_{1}$ and $\Gamma_{2}$ are conjugate, then the manifolds are isometric.)

Before stating our first theorem, we recall that any Lie group $G$ which admits a uniform discrete subgroup is unimodular. For $\gamma \in \Gamma$, the centralizer $C(\gamma, \Gamma)$ is a uniform discrete subgroup of the centralizer $C(\gamma, G)$, so $C(\gamma, G)$ is also unimodular. Given any conjugacy class of unimodular subgroups of $G$, we can define a Haar measure on each subgroup in such a way that if $\mathrm{K}=a \mathrm{Ha}^{-1}$, then the conjugation by $a$ is a measure-preserving transformation from $H$ to $K$. In the following theorem, we assume Haar measures have been so chosen on $C\left(\gamma_{i}, G\right)$. For any group $H$ and any element $h \in H$, we denote by $[h]_{H}$ the conjugacy class of $h$ in $H$, and we denote by $[H]$ the set of all conjugacy classes of elements of $H$, so $[h]_{H} \in[H]$.

Received by the editors July 1, 1986.

1980 Mathematics Subject Classification (1985 Revision). Primary 58G25, 53C20.

First author supported by the Sloan Foundation and NSF Grant DMS 85-03302; second author supported by NSF Grant DMS 85-02084. 
THEOREM 1. Suppose that a Lie group $G$ acts freely by isometries on a connected Riemannian manifold $(M, g)$, that the discrete subgroups $\Gamma_{1}$ and $\Gamma_{2}$ of $G$ act properly discontinuously and that $\Gamma_{i} \backslash M$ is compact for $i=1,2$. If for all $[h]_{G} \in[G]$ we have

(*)

$$
\sum_{\left[\gamma_{1}\right]_{\Gamma_{1}} \subset[h]_{G}} \operatorname{vol}\left(C\left(\gamma_{1}, \Gamma_{1}\right) \backslash C\left(\gamma_{1}, G\right)\right)=\sum_{\left[\gamma_{2}\right]_{\Gamma_{2}} \subset[h]_{G}} \operatorname{vol}\left(C\left(\gamma_{2}, \Gamma_{2}\right) \backslash C\left(\gamma_{2}, G\right)\right),
$$

then $\left(\Gamma_{1} \backslash M, g\right)$ is isospectral to $\left(\Gamma_{2} \backslash M, g\right)$.

The condition $(*)$ reduces to Sunada's condition when $G$ is finite. The conclusion of the theorem can be strengthened in the sense that the manifolds are isospectral in a stronger sense than that defined above: the associated Laplacians acting on $p$-forms are also isospectral for every $p=0,1, \ldots, \operatorname{dim} M$. The hypotheses of Theorem 1 can also be weakened slightly (details will be given in [DG2]). With the exception of certain Heisenberg manifolds for which the associated Laplacians are isospectral on functions but not on 1-forms, all known examples of closed isospectral manifolds satisfy at least the weakened hypotheses.

EXAMPLE. In [GW and DG1], continuous families of isospectral manifolds $\left(\Gamma_{t} \backslash M, g\right)$ for $t \in \mathbf{R}$ are constructed. In such a family, each pair of manifolds satisfies the hypotheses of Theorem 1. The group $G$ is assumed to be a nilpotent Lie group for which there exists a one-parameter group of automorphisms $\Phi_{t}$ (which are noninner except for perhaps a discrete subset of $t$ 's) such that $\Gamma_{t}=\Phi_{t}\left(\Gamma_{0}\right)$ and $\Phi_{t}(\gamma)$ is conjugate in $G$ to $\gamma$ for all $\gamma \in \Gamma_{0}$. The verification of condition (*) reduces to the observation that the $\Phi_{t}$ 's are necessarily volume-preserving. Specific examples are given in the papers cited above. Theorem 1 actually allows us to remove the hypothesis in [DG1] that $G$ be two-step nilpotent.

We next turn to the problem of constructing isospectral, noncongruent potentials. Until recently, the only examples known were continuous families constructed by means of the Korteweg-deVries equation or its higher-order analogues. Then Brooks [B] showed how to modify Sunada's technique to produce pairs of isospectral potentials. If $\tau \in I(M)$, a potential function $u$ is said to be $\tau$-invariant if $\tau^{*} u=u$. In particular, if $u$ is $\Gamma$-invariant, then $u$ defines a potential, again denoted $u$ on $\Gamma \backslash M$. Brooks observed that, under Sunada's hypotheses, if $u$ is a $G$-invariant potential, then the operators $\Delta_{1}+u$ on $L^{2}\left(\Gamma_{1} \backslash M\right)$ and $\Delta_{2}+u$ on $L^{2}\left(\Gamma_{2} \backslash M\right)$ have the same spectra (where $\Delta_{i}$ is the Laplacian of $\left.\Gamma_{i} \backslash M\right)$. If coincidentally there exists $\tau \in I(M) \backslash G$ such that $\Gamma_{2}=\tau \Gamma_{1} \tau^{-1}$ (and hence $\left(\Gamma_{1} \backslash M, g\right)$ is isometric to $\left.\left(\Gamma_{2} \backslash M, g\right)\right)$ but $\tau^{*} u \neq u$, then $\tau^{*}\left(\Delta_{2}+u\right)=\Delta_{1}+\tau^{*} u$ has the same spectrum as $\Delta_{1}+u$; i.e., $u$ and $\tau^{*} u$ are isospectral potentials on $\left(\Gamma_{1} \backslash M, g\right)$. By choosing $u$ carefully, one can arrange that $u$ and $\tau^{*} u$ are not congruent. A similar argument yields

THEOREM 2. Assume the hypotheses of Theorem 1 and assume moreover that there exists $\tau \in I(M) \backslash G$ such that $\Gamma_{2}=\tau \Gamma_{1} \tau^{-1}$. Let $u$ be a G-invariant potential function on $M$ which is not $\tau$-invariant. Then $u$ and $\tau^{*} u$ are isospectral potentials on $\left(\Gamma_{1} \backslash M, g\right)$. 
To obtain continuous families of isospectral potentials, we begin with a nilpotent Lie group $\tilde{G}$, uniform discrete subgroups $\tilde{\Gamma}_{t}$ and automorphisms $\Phi_{t}$ as in the example above. Let $H=\mathbf{R} \cdot \tilde{G}$ be the semidirect product, where $t \in \mathbf{R}$ acts on $\tilde{G}$ as the automorphism $\Phi_{t}$. We will denote elements of $H$ as ordered pairs $(t, x)$ with $t \in \mathbf{R}$ and $x \in \tilde{G}$. We require that for some $a>0$, $\Phi_{a}\left(\tilde{\Gamma}_{0}\right)=\tilde{\Gamma}_{0}$ (i.e., $\tilde{\Gamma}_{a}=\tilde{\Gamma}_{0}$ ), and we let $a_{0}$ be the minimum such number. We then have that $\Phi_{n a_{0}}\left(\tilde{\Gamma}_{t}\right)=\tilde{\Gamma}_{t}$ for all $t \in \mathbf{R}, n \in \mathbf{Z}$ and hence $\Gamma_{t} \equiv a_{0} \mathbf{Z} \cdot \tilde{\Gamma}_{t}$ is a uniform discrete subgroup of $H$. Let $M=H$ with a left-invariant metric $g$, and let $G=\Gamma_{o} \tilde{G}\left(=\Gamma_{t} \tilde{G}\right)$. Then one can check for each $t \notin a_{0} \mathbf{Z}$ that $M, G, \Gamma_{0}$ and $\Gamma_{t}$ satisfy the hypotheses of Theorem 2 with $\tau=\tau_{t}$ given by left multiplication by $(t, 1)$. Let $v$ be any smooth periodic function on $\mathbf{R}$ of period $a_{0}$ and define $u(s, x)=v(s)$. Then $\tau_{t}^{*} u(s, x)=u(s+t, x)$. This defines a family of isospectral potentials on $\left(\Gamma_{0} \backslash H, g\right)$. By choosing $u$ carefully, one can arrange that the deformation is nontrivial.

Details and examples of all the results above and generalizations to manifolds with boundary will be given in [DG2].

\section{REFERENCES}

[B] R. Brooks, On manifolds with negative curvature with isospectral potentials, preprint, 1985.

[DG1] D. DeTurck and C. Gordon, Isospectral deformations I: Riemannian structures on two-step nilspaces, Comm. Pure Appl. Math. 40 (1987), 367-387.

[DG2] _ Isospectral deformations II: Metrics and potentials on general manifolds, preprint, 1987.

[GW] C. Gordon and E. Wilson, Isospectral deformations of compact solvmanifolds, J. Differential Geom. 19 (1984), 241-256.

[S] T. Sunada, Riemannian coverings and isospectral manifolds, Ann. of Math. (2) 121 (1985), 169-186.

Department of Mathematics,University of Pennsylvania, PhiladelPHIA, PENNSYLVANIA 19104

Department of Mathematics, Washington University, Saint LoUis, MisSOURI 63130 
